\title{
Rejecting Astrophysical False Positives from the TrES Transiting Planet Survey: The Example of GSC 03885-00829
}

\section{Citation}

O'Donovan, Francis T., David Charbonneau, Guillermo Torres, Georgi Mandushev, Edward W. Dunham, David W. Latham, Roi Alonso, et al. 2006. "Rejecting Astrophysical False Positives from the TrES Transiting Planet Survey: The Example of GSC 03885-00829." The Astrophysical Journal 644 (2): 1237-45. https://doi.org/10.1086/503740.

\section{Permanent link}

http://nrs.harvard.edu/urn-3:HUL.InstRepos:41397403

\section{Terms of Use}

This article was downloaded from Harvard University's DASH repository, and is made available under the terms and conditions applicable to Other Posted Material, as set forth at http:// nrs.harvard.edu/urn-3:HUL.InstRepos:dash.current.terms-of-use\#LAA

\section{Share Your Story}

The Harvard community has made this article openly available.

Please share how this access benefits you. Submit a story.

\section{Accessibility}


Submitted 7 November 2005; accepted 27 February 2006

Preprint typeset using LATEX style emulateapj v. 6/22/04

\title{
REJECTING ASTROPHYSICAL FALSE POSITIVES FROM THE TrES TRANSITING PLANET SURVEY: THE EXAMPLE OF GSC 03885-00829 ${ }^{1}$
}

Francis T. O'Donovan ${ }^{2}$, David Charbonneau ${ }^{3}$, Gulllermo Torres $^{3}$, Georgi Mandushev $^{4}$, Edward W. Dunham ${ }^{4}$, David W. Latham ${ }^{3}$, Roi Alonso ${ }^{5}$, Timothy M. Brown ${ }^{6}$, Gilbert A. Esquerdo ${ }^{7}$, Mark E. Everetr ${ }^{7}$,

Orlagh L. Creevey 6

Submitted 7 November 2005; accepted 27 February 2006

\begin{abstract}
Ground-based wide-field surveys for nearby transiting gas giants are yielding far fewer true planets than astrophysical false positives, of which some are difficult to reject. Recent experience has highlighted the need for careful analysis to eliminate astronomical systems where light from a faint eclipsing binary is blended with that from a bright star. During the course of the Trans-atlantic Exoplanet Survey, we identified a system presenting a transit-like periodic signal. We obtained the proper motion and infrared color of this target (GSC 03885-00829) from publicly available catalogs, which suggested this star is an F dwarf, supporting our transit hypothesis. This spectral classification was confirmed using spectroscopic observations from which we determined the stellar radial velocity. The star did not exhibit any signs of a stellar mass companion. However, subsequent multi-color photometry displayed a color-dependent transit depth, indicating that a blend was the likely source of the eclipse. We successfully modeled our initial photometric observations of GSC 03885-00829 as the light from a K dwarf binary system superimposed on the light from a late F dwarf star. High-dispersion spectroscopy confirmed the presence of light from a cool stellar photosphere in the spectrum of this system. With this candidate, we demonstrate both the difficulty in identifying certain types of false positives in a list of candidate transiting planets and our procedure for rejecting these imposters, which may be useful to other groups performing wide-field transit surveys.
\end{abstract}

Subject headings: stars: binaries: eclipsing — stars: planetary systems - techniques: photometric techniques: radial velocities — stars: individual: alphanumeric: GSC 03885-00829

\section{INTRODUCTION}

The discovery of the 169 known extrasolar planets 8 has greatly enhanced our understanding of planetary systems. Most of these extrasolar planets have been identified from Doppler surveys, which search for the radial velocity variation of a star caused by the presence of a gas giant. These observations can estimate the period and eccentricity of the planetary orbit, and provide a lower limit for the planetary mass relative to that of the star. Assuming that the stellar mass can be precisely determined, perhaps by comparing the stellar spectra to stellar atmosphere models (such as Kurucz 1993), the minimum mass of the planet can be derived. The diversity of these planetary systems has drastically altered our the-

\footnotetext{
${ }^{1}$ Some of the data presented herein were obtained at the W.M. Keck Observatory, which is operated as a scientific partnership among the California Institute of Technology, the University of California, and the National Aeronautics and Space Administration. The Observatory was made possible by the generous financial support of the W.M. Keck Foundation.

${ }^{2}$ California Institute of Technology, 1200 East California Boulevard, Pasadena, CA 91125; ftod@caltech.edu

3 Harvard-Smithsonian Center for Astrophysics, 60 Garden Street, Cambridge, MA 02138

${ }^{4}$ Lowell Observatory, 1400 West Mars Hill Road, Flagstaff, AZ 86001

${ }^{5}$ Instituto de Astrofísica de Canarias, 38200 La Laguna, Tenerife, Spain

6 High Altitude Observatory/National Center for Atmospheric Research, 3080 Center Green, Boulder, CO 80301

7 Planetary Science Institute, 1700 East Fort Lowell Road, Suite 106, Tucson, AZ 85719

${ }^{8}$ Updates available from the Extrasolar Planets Encyclopaedia: http://www.obspm.fr/planets/
}

oretical appreciation of their morphology and evolution, including the existence of "hot Jupiters", Jupiter-sized planets with periods of a few days that experience high insolation from the nearby star. However, less is known about the nature of the planets themselves, except for the cases when a hot Jupiter was observed to pass in front of a dwarf star. By observing such a transit (as was first suggested by Struve 1952), we can estimate the radius of the transiting planet from the fraction of starlight the planet blocks during the transit and the radius of the star itself, which must be measured from stellar model fits to spectra of the star. For a transit to occur, the planetary orbital inclination must be approximately $90^{\circ}$; this implies that the Doppler limit for the planetary mass must be close to the actual mass of the planet. We now have estimates for the radii and masses of 9 planets from transit observations similar to those first successfully performed by Charbonneau et al. (2000) and Henry et al. (2000). These estimates provide constraints for the competing theories of planetary formation. For example, the recently discovered transiting "hot Saturn" HD $149026 \mathrm{~b}$ has a mass and radius that imply the planet has a large core (approximately $70 \mathrm{M}_{\oplus}$; Sato et al. 2005; Charbonneau et al. 2006). It is hypothesized that this planet must have formed via core accretion (Pollack 1984; Pollack et al. 1996), rather than through gravitational instability (Boss 1997). However, not every observation of a transiting planet can be explained by current models of close-in giant planets experiencing high stellar insolation. The transiting planet HD 209458 b has a radius larger than other planets of its mass, and larger than the 
predicted radii (see Laughlin et al. 2005, and references therein, and Deming et al. 2005).

Several wide-field photometric surveys with the goal of identifying nearby transiting planets are currently active. Our Trans-atlantic Exoplanet Survey ${ }^{9}$ (TrES) is a network of three $10 \mathrm{~cm}$ telescopes: Sleuth (located at Palomar Observatory, California), PSST (Lowell Observatory, Arizona; Dunham et al.|2004), and STARE ${ }^{10}$ (Tenerife, Spain; Alonso et al. 2004b). The TrES campaign, together with other wide-field surveys such as the HAT network (Bakos et al. 2002) and SuperWASP (Street et al. 2003), are monitoring thousands of nearby bright stars $(9 \leq V \leq 13)$. We hope to find recurring eclipses with the short period and small amplitude corresponding to a transiting hot Jupiter. The brightness of the target stars facilitates both the photometric precision and the follow-up of any identified transiting planets using space-borne telescopes. Examples of detailed followup observations include the measurement of several chemical abundances in the atmosphere of HD $209458 \mathrm{~b}$ (Charbonneau et al. 2002; Vidal-Madiar et al. 2003) and the first direct detections of emitted planetary radiation (Charbonneau et al. 2005; Deming et al. 2005).

Many astrophysical systems exist that mimic the light curve of a transiting hot Jupiter. Due to the mass-radius degeneracy for bodies with masses between 0.001 and $0.1 \mathrm{M}_{\odot}$, the depth of an eclipse of a solar-type star by a hot Jupiter, a brown dwarf or an M dwarf star will be about $1 \%$ in each case, despite the large range in mass. Grazing incidence eclipsing binaries may also exhibit comparable eclipse depths. For wide-field ground-based surveys, the frequency of the false positives is greater than the frequency of detection of true transiting planets, by at least an order of magnitude. As part of TrES, we typically identify $10-20$ of these transit-like photometric signals out of $15,000-25,000$ stars $(10<V<15)$ in each $6^{\circ} \times 6^{\circ}$ target field of view $\left(b \sim 15^{\circ}\right)$ (see, e.g., Dunham et al. 2004), and similar yields should be expected from other wide-field ground-based surveys. This number (which is dependent on the density of star counts, and hence the Galactic latitude) is consistent with theoretical predictions. For example, Brown (2003) predicts that for every 25,000 stars observed, we will find 10 false positives and only one true transiting planet. This assumes that we must observe 3 transit events for each candidate, and is dependent on the visibility of transits throughout the observation run. The low yield of planets necessitates a rigorous routine of follow-up observations and detailed analysis to eliminate all possible alternatives to the planet hypothesis. One straightforward method to reduce the number of false positives is to obtain multi-epoch spectroscopy of each candidate and measure radial velocities with a precision of $\sim 1 \mathrm{~km} \mathrm{~s}^{-1}$. From this we can identify targets with companions of stellar, rather than planetary, mass (see, e.g., Latham 2003; Charbonneau et al. 2004). We can also estimate the luminosity class of the target star to single out and reject giants.

The blending of the light from an edge-on binary system with that from a third star can also be mistaken for a transit. Brown (2003) predicts that half of the

\footnotetext{
9 http://www.astro.caltech.edu/ ftod/tres/

10 http://www.hao.ucar.edu/public/research/stare/stare.html
}

false positives from a typical wide-field survey will be of this type; the other half will be grazing eclipsing binaries. Blends can be much more difficult to identify. The faintness of the binary compared to the third star can prevent the detection of the radial velocity variations of the binary. These variations are also masked by the rotationally broadened spectral lines of the rotationally synchronized binary stars. However, if the binary has a significant difference in effective temperature from that of the third star, the eclipse depths should display a strong color dependence, unlike the color-independent transits of a (dark) planet across a single star. Nevertheless, there has been recent experience of blends with color-independent eclipse depths. In the case of OGLE-TR-33 (Torres et al. 2004b) and GSC 01944-02289 (Mandushev et al. 2005), both candidates (a suspected planet and brown dwarf, respectively) showed color-independent eclipse depths, and yet were subsequently discovered to be blended systems. Evidence for the presence of an eclipsing binary was found from a careful analysis of the spectral line shapes, prompting the authors to compare the photometric data to simulations of blends. OGLE-TR-33 was shown to be a hierarchical triple consisting of a bright F6 dwarf and an F4+(K7M0) binary. The blend model for GSC 01944-02289 comprises an F5 primary and a G0+M3 binary. In both cases, the similarity in color between the primary star and the brightest member of the binary explains the constant eclipse depth at different wavelengths. The high occurrence of such false positives and the difficulty in rejecting them requires a detailed study of candidates before any announcement is made, as was done in the case of TrES-1 (Alonso et al. 2004a) and OGLE-TR-56 b (Torres et al. 2004a).

Here we discuss a promising candidate, GSC 03885-00829, from one of our target fields. Initial photometric (\$2) and spectroscopic (\$3) monitoring of this candidate strongly suggested that we were observing a Saturn-sized companion transiting a solar type star every 1.441 days with a transit depth of approximately 6 mmag. However, follow-up photometry (\$) displayed a slight color dependence as might be caused by a blend, and we were able to model our photometry using simulations of blended eclipsing binaries (5. Our best fit model consists of a bright $\mathrm{F}$ dwarf and a K dwarf binary, and we were able to identify the presence of light from the binary in the spectrum of GSC 03885-00829 (6). The faintness of the binary system prevents us from identifying the presence of asymmetric spectral lines, as was done by Torres et al. (2004b) and Mandushev et al. (2005). In this case, only multi-color observations provided us with the necessary evidence to call into question the planetary nature of this candidate.

\section{TRES TELESCOPE OBSERVATIONS}

In March 2004, we commenced observations of a $6^{\circ} \times 6^{\circ}$ target field in Draco. The field is centered on our $V=4.8$ guide star HD $151613\left(\alpha=16^{\mathrm{h}} 45^{\mathrm{m}} 17^{\mathrm{s}} 82\right.$, $\delta=+56^{\circ} 46^{\prime} 54^{\prime \prime} .7$ J2000). Between UT 2004 March 29 and June 22, we observed this field nightly with Sleuth at Palomar Observatory (California), and with PSST at Lowell Observatory (Arizona). STARE, in Tenerife (Spain), did not observe this field as it was undergoing 
repairs at this time. A total of 15854 photometric exposures of $90 \mathrm{~s}$ each were obtained through either a Sloan $r$ filter (Sleuth) or a Kron-Cousins $R$ filter (PSST).

We bias-subtracted and flat-fielded the images of our target field once the data were transferred from the observatory computers. We performed the calibration of the Sleuth data using customized IDL routines; we calibrated the PSST data using the zerocombine, ccdproc, and flatcombine tasks in the IRAF $^{11}$ package (Todv 1993). We reduced the Sleuth and PSST photometric data separately as follows using our difference image analysis (DIA) pipeline (described in Dunham et al. 2004, and based in part upon Alard 2000).

We created our reference image for the field from images obtained at low air mass on a photometric night during dark time. We obtained a standard list of stars from this image using profile-fitting (PSF) photometry (DAOPHOT II/ALLSTAR; Stetson 1987, 1992). We calculated the equatorial coordinates $(\alpha, \delta)$ of these stars by matching a subset with the stars listed in the Tycho2 Catalog (Høg et al. 2000), and then spatially interpolated all of the science images so that the star coordinates from each image matched those from our standard star list.

We produced the master image for the Sleuth data set by combining 19 of our best-quality interpolated images; we combined 17 images to create the corresponding PSST master image. We subtracted each interpolated image from this master image. We used aperture photometry on the resultant difference images (using the centroids derived for the standard star list) to estimate the flux of each star in each image. We produced time series consisting of the differences between the magnitude of a star in the reference image and the magnitude of that star in each target image in turn. We decorrelated these light curves as follows to remove systematic effects typical of wide-field surveys, such as those caused by changing atmospheric conditions throughout the night. We listed the stars in order of brightness, and divided the list into batches of 500. We computed the least-squares fit to the light curve of a given star from a linear combination of the other light curves in that batch. We then subtracted this least-squares fit from the light curve of the star.

In our previous studies (Alonso et al. 2004a; Mandushev et al. 2005; Creevev et al. 2005), we presented separate light curves from one of the TrES telescopes. For this field, we combined the two data sets. For a given star on the Sleuth standard star list, we calculated the angular distances between that star and the PSST standard stars. We matched the Sleuth star with a PSST star if the angular distance was less than $5^{\prime \prime}$ (0.5 pixels). Due to the difference in the selected filter and the telescope pointing between the two sites, some stars were unique to a given standard star list, and no match was found. We appended the time series for each matched PSST star to the corresponding Sleuth time series, and reordered the combined time series chronologically. The data for the unmatched PSST stars were simply added to the resultant data set.

11 IRAF is distributed by the National Optical Astronomy Observatories, which are operated by the Association of Universities for Research in Astronomy, Inc., under cooperative agreement with the National Science Foundation.
In order to reduce the computational intensity of our transit search, we averaged the combined time series in 9 minute wide bins to obtain 2996 binned observations. Since central transits should last 3 hours, this did not significantly sacrifice temporal resolution of potential transit events. For $\sim 10,000$ stars, the rms scatter of the binned data was below $0.04 \mathrm{mag}$. We performed a search of the time series of these stars using the box-fitting least squares transit-search algorithm (BLS; Kovács. Zucker, \& Mazeh 2002) to identify periodic transit events. The BLS algorithm calculates a Signal Detection Efficiency (SDE; see Kovács et al. 2002) for each candidate, which denotes how significant the detection is. We identified candidates based on this SDE, followed by a visual inspection.

Many of the candidates identified for this field show V-shaped eclipses or a possible ellipsoidal variability, or have large depths, making it likely that they are not transiting planets, but rather eclipsing binaries. However, we promptly identified a promising candidate. When the data for this star were folded with the photometric orbital period of 1.441 days (calculated using the BLS algorithm), the resultant light curve (Figure 1) displayed a shallow and flat-bottomed transit, and no noticeable ellipsoidal variability out of transit. The depth ( $\sim 6 \mathrm{mmag})$ and duration (1.4 hours) of the occultation are consistent with a Saturn-sized planet transiting a solar type star. The SDE for this candidate $(\sim 20)$ was high relative to that calculated for typical TrES candidates $(\sim 10-15)$.

Supporting data from online catalogs provided further evidence of the planetary nature of this eclipse. Using the SIMBAD ${ }^{12}$ database, we identified our candidate as the star GSC 03885-00829 (see Table 1). The infrared colors (2MASS $J-K, J-H$; Cutri et al. 2003) and optical $(B-V)$ colors of this star are near-solar, roughly consistent with the stellar parameters inferred from our transit observations. This star displays significant proper motion (26 mas/year from the USNO-B Catalog; Monet et al. 2003), suggesting it is a nearby dwarf. As a first check of the possibility of contamination of light from a nearby star, we verified that there is no bright star visible on the Digitized Sky Survey ${ }^{13}$ (DSS) images within our aperture radius $\left(\leq 30^{\prime \prime}\right)$.

With due enthusiasm, we proceeded to obtain followup observations of this exciting candidate, with the goal of rejecting the possibility that this was not a transiting planet.

\section{SPECTROSCOPIC FOLLOW-UP}

We confirmed that GSC 03885-00829 was an isolated dwarf star by spectroscopically monitoring this candidate. We observed GSC 03885-00829, together with other candidates from this TrES field, with the Harvard-Smithsonian Center for Astrophysics (CfA) Digital Speedometer (Latham 1992), operated on the $1.5 \mathrm{~m}$ Tillinghast reflector at the F. L. Whipple Obser-

\footnotetext{
12 http://simbad.harvard.edu/

13 The Digitized Sky Survey /http://archive.stsci.edu/dss/ was produced at the Space Telescope Science Institute under U.S. Government grant NAG W-2166. The images of these surveys are based on photographic data obtained using the Oschin Schmidt Telescope on Palomar Mountain and the UK Schmidt Telescope. The plates were processed into the present compressed digital form with the permission of these institutions.
} 
vatory (FLWO) on Mt. Hopkins, Arizona. The spectral coverage was $45 \AA$ centered on $5187 \AA$ at a resolving power of $\lambda / \Delta \lambda \approx 35,000$ (a resolution of $8.5 \mathrm{~km} \mathrm{~s}^{-1}$ ). We observed this particular target on UT 2005 May 18, May 20 and May 21, at an orbital phase $0.52,0.86$, and 0.50 , respectively (calculated using the orbital ephemeris of the planet; see Table 1 ).

Radial velocities were obtained by cross-correlation using templates chosen from a library of synthetic spectra computed for us by J. Morse and based on the model atmospheres of R. L. Kurucz (J. Morse \& R. L. Kurucz, 2004 , private communication). The typical precision of a single velocity measurement is $0.5 \mathrm{~km} \mathrm{~s}^{-1}$. We measured the radial velocity to be constant $\left(-38.48 \mathrm{~km} \mathrm{~s}^{-1}\right.$ with an rms of $0.28 \mathrm{~km} \mathrm{~s}^{-1}$ ) within our errors. These measurements indicate that the target star is not gravitationally bound to a massive stellar companion. Various stellar parameters were estimated, again by crosscorrelating these spectra against a grid of templates from our spectral library, seeking the best match. Assuming a solar metallicity, we estimated the effective temperature to be $T_{\text {eff }}=6150 \mathrm{~K}$ and the surface gravity to be $\log g \approx 4.4$, which suggested this was a late $\mathrm{F}$ dwarf star, consistent with the proper motion and photometric colors, and with our transit hypothesis. The formal stellar rotation we derived $\left(v \sin i \approx 1 \mathrm{~km} \mathrm{~s}^{-1}\right)$ is actually below our spectral resolution. The surface gravity suggests the star is unevolved. This constraint is important, as this star lies within the range of effective temperatures for which an ambiguity exists as to the corresponding mass of the star while on the main sequence, depending on the degree of evolution. An illustration of this ambiguity is shown in Figure 3. where the two locations denoted by the open circle and large filled circle have the same effective temperature as GSC 03885-00829 but rather different luminosities. The particular age of the isochrone for this figure was selected to show this difference more clearly. The fainter (lower) location corresponds to an unevolved main-sequence star $(\log g=4.34)$ of mass $1.15 \mathrm{M}_{\odot}$, whereas the brighter location is for a star near the end of the hydrogen-burning phase, and has a surface gravity of $\log g=3.68$ and a mass of $1.61 \mathrm{M}_{\odot}$. The radii differ by a factor of about 2.4. From this example, we see that, without a surface gravity constraint, we cannot be sure of the radius of our target star, preventing our discriminating between a planetary and a stellar transiting companion.

\section{PHOTOMETRIC FOLLOW-UP}

Although we had found no evidence of a stellar mass companion from our radial velocity measurements, the possibility remained that the observed transits were in fact the eclipses of a faint binary whose light was blended with that from the bright $\mathrm{F}$ dwarf due to the large pixel sizes of our detectors. Follow-up observations of higher angular resolution might resolve such a blended system. A possible wavelength dependence of the eclipse depth from multi-color observations would also provide evidence of a blend. We organized a followup photometric campaign to observe multiple transits of GSC 03885-00829 using $D>20^{\prime \prime}(0.50 \mathrm{~m})$ telescopes and through several different filters.

High precision photometry of GSC 03885-00829 was made on UT 2005 June 8 using the $1.2 \mathrm{~m}$ FLWO tele- scope (Arizona). For this clear, photometric night, we used MiniCam, a two CCD mosaic, each array being $2048 \times 4608$ pixels. Observations were binned $2 \times 2$ for a faster duty cycle. A Sloan $g$ filter was used, with an exposure time of $30 \mathrm{~s}$ and a corresponding duty cyle of $\sim 50.5 \mathrm{~s}$. The telescope was de-focused to a FWHM of $\sim 15$ pixels $\left(\sim 9^{\prime \prime}\right)$ to allow greater photon counts per given exposure without saturation. Spreading the star image also serves to reduce pixel-to-pixel variations that may not be completely removed by flat-fielding. A total of 561 photometric measurements of the field were made over a total of 7.875 hours. The differential light curve of our target star was obtained using aperture photometry of this star and one of the observed reference stars. We corrected for the effect of differential extinction on the time series by fitting the out-of-eclipse data. The resultant light curve was then converted to flux units (see Figure 4). These follow-up photometric observations were made over a year after our TrES images. We used this separation in time to obtain a more accurate photometric ephemeris for our candidate, $T_{c}($ HJD $)=2453529.833+1.44122 \times E$ (see Table 1).

GSC 03885-00829 was observed on the night of UT 2005 June 13 at the $0.82 \mathrm{~m}$ IAC-80 telescope at the Observatorio del Teide (Tenerife, Spain), using its $1024 \times 1024$ pixels CCD and a Johnson $R$ filter. To achieve better photometric precision, a slight defocus was applied, so as to image stars with a FWHM of $\sim 4^{\prime \prime}$. Exposures times of $22 \mathrm{~s}$ were used, and the readout time using a $2 \times 2$ binning was $\sim 10 \mathrm{~s}$. The images were biasand flat-field corrected, and aperture photometry was applied using the package for optimal aperture photometry vaphot (Deeg \& Dovle 2001). Nine reference stars were used to build an essemble reference star. The dispersion of the data points is larger at the end of the night as the star was closer to the horizon. We corrected for differential extinction; Figure 4 shows the derived differential light curve.

We obtained $B V(R I)_{\mathrm{C}}$ observations of GSC $03885-00829$ on UT 2005 June 5 with the Lowell Observatory $42^{\prime \prime}(1.05 \mathrm{~m})$ Hall reflector in combination with a $2 \mathrm{~K} \times 2 \mathrm{~K}$ SITe CCD. A total of 223 exposures of the program field were accumulated: four in $B, 211$ in $V$, three in $R_{C}$, and five in $I_{C}$. We observed 20 photometric standards in the SA107, SA108, SA109, SA112, and PG1633 fields (Landolt 1992) in order to calibrate the photometry. We obtained the following values ${ }^{14}$ for the standard magnitudes of GSC 03885-00829 (the numbers in the brackets show the number of individual measurements used to derive the mean magnitudes): $B=11.077 \pm 0.001$ (4), $V=10.465 \pm 0.001$ (12), $R_{\mathrm{C}}=10.094 \pm 0.003(3)$, and $I_{\mathrm{C}}=9.722 \pm 0.001$ (5).

These initial attempts to observe GSC 03885-00829 convinced us that the target star displayed a transitlike dip and that nearby stars were not variable. This reduced the possibility that this signal was caused by a chance superposition of a star with an eclipsing binary in the large pixel scale of the TrES detectors. We were also able to reproduce our $g$ band observations using a model of a Jupiter-sized planet orbiting a near-solar type star,

14 The errors include the uncertainties in the Landolt photometry and the internal scatter of our photometry, but may not account for the total systematic error in the observations. 
although the ingress and egress appeared to be too long in duration.

It was when we compared the different light curves that we realized something was wrong with our assumption that these were observations of a transiting planet. The depth of the transit appeared to vary with wavelength: an eclipse depth of $0.4 \%$ in the $g$ band and $0.7 \%$ in the $R$ band. Prompted by the color dependence of the transit depths, we ran various simulations of the eclipses visible from GSC 03885-00829 in an attempt to rule out the possibility of a blend.

\section{BLEND ANALYSIS}

Light curve fits to the $g$-band observations were carried out as described in detail by Torres et al. (2004b). Briefly, we assumed that the measured brightness of GSC 03885-00829 is due to the light of an eclipsing binary blended with the light of the F star, so that the deep eclipses of the binary are reduced in depth to the level that we see. We hypothesized that the three objects formed a hierarchical triple system (rather than a bychance alignment), and we took their physical properties from theoretical isochrones by Girardi et al. (2000). The mass of the $\mathrm{F}$ star $\left(1.15 \mathrm{M}_{\odot}\right)$ was constrained from its effective temperature derived in $\$ 3$ with the assumption that it is unevolved. This modeling produced a reasonably good match to the measured dip for an edge- on binary composed of an early $K$ star eclipsed by a very small $\mathrm{M}$ star (see Figure 5). In this scenario there is no measurable secondary eclipse. Although the eclipse depth is well reproduced, the predicted duration is slightly shorter than that observed. In addition, the brightness expected for the K star ( $\sim 15 \%$ of the F star in the optical) is such that it would be visible in our spectra. This fit could only be improved by increasing the size (and mass) of the brightest star to a value inconsistent with the surface gravity inferred from our spectroscopy.

The periods for our transit candidates are calculated assuming each observed transit is of equal depth. However, when a candidate is in fact a blended eclipsing binary, the possibility exists that the primary and secondary eclipses are similar enough in depth to be confused by our period-finding technique. The BLS algorithm may derive a best fit period for such a system that is half of the true value. Therefore, we explored blend scenarios in which the period is $2 \times 1.441$ days. Figure 6 shows the result of our best fit to the TrES $r$-band data, in which the eclipsing binary is composed of two late $\mathrm{K}$ stars with masses of $0.67 \mathrm{M}_{\odot}$ and $0.64 \mathrm{M}_{\odot}$, with an orbital inclination of $84^{\circ}$ to the line of sight. The model indicates a slight difference in eclipse depths (see Figure 6a) of about $1 \mathrm{mmag}$, although this difference is only marginally visible in observations themselves. Enlargements of the two eclipse regions are displayed in the lower panels of Figure 6. According to this fit, the time of the center of transit previously derived (see Table1) is in fact a time of secondary eclipse; all of the photometric follow-up observations shown in Figure 4 were taken during a secondary eclipse. The brighter of the $\mathrm{K}$ stars (i.e., the primary of the eclipsing binary) has only $\sim 3 \%$ of the light of the main F star in the optical, and is below our threshold for spectroscopic detection. Figure 3 shows the location of the three stars (the filled circles) in the $\mathrm{H}-\mathrm{R}$ diagram.
According to the blend model described above, the eclipse in the $g$ band (a secondary eclipse) is predicted to be shallower than in the $r$ band $(0.35 \%$ versus $0.7 \%)$, as we indeed observe, although the measured depth (0.4\%) is slightly deeper than predicted. We attribute this to shortcomings in the isochrones used for the blend modeling, which are not specifically designed for low-mass stars. In particular, missing opacity sources and other physical ingredients may affect the theoretical luminosities in the optical ( $V$ or $g$ ) bands (Baraffe et al. 1998; Delfosse et al. 2000; Chabrier et al. 2005), whereas the red and near infrared magnitudes are presumably more reliable. A sign of this is seen perhaps in the predicted $V-K$ color for the main $\mathrm{F}$ star: the isochrones give $V-K=1.26$ (in the Johnson system as defined by Bessell \& Brett 1988), bluer than the typical color of a dwarf of this temperature, $V-K=1.40$ (e.g., Bessell \& Brett 1988). Other stellar evolution models specifically designed for low-mass stars such as those by Baraffe et al. (1998) appear to give more realistic colors. Our $\mathrm{F}$ star is predicted to have $V-K=1.41$ according to those calculations (after transformation of the isochrone $K$ magnitudes from the CIT to the Johnson system, following Leggett 1992), very close to the empirical value. Unfortunately the Baraffe et al. (1998) models are not publicly available for the Sloan bands, so we are unable to use them in our blend modeling.

As indicated earlier, infrared magnitudes for GSC 03885-00829 are available from the 2MASS Catalog. In particular, the measured $V-K$ color of our candidate in the Johnson system (Table 1) is $1.66 \pm 0.02$ (using transformations from the 2MASS system by Carpenter 2001). The difference with the color of a single $\mathrm{F}$ star indicates a significant infrared excess of about a quarter of a magnitude ${ }^{15}$. This in itself can be taken as evidence of contamination from the light of a later-type object, providing further evidence that we are dealing with a blend. The computed color of the combined light of the three stars in our model using the Baraffe et al. (1998) isochrones is $V-K=1.64$, which agrees quite well with the observations and supports our interpretation. Other predicted red and infrared colors also match the measured values reasonably well (see Figure (7).

Although it is quite possible that additional finetuning may improve the small discrepancies noted above in the $g$ band and provide a near-perfect fit to all observations (to the extent allowed by the accuracy of the stellar evolution models and observational uncertainties), our goal here has been to show how subtle the signatures of a blend can be, and that with careful modeling it is possible to demonstrate that they are in fact due to a blend scenario, and therefore to reject the candidate.

\section{CONFIRMATION OF BLEND MODEL}

In order to test further our blend hypothesis, we observed GSC 03885-00829 on UT 15 August 2005 with the NIRSPEC infrared spectrograph at the Keck Observatory. We observed the target in the $K$-band spectral region centered near $2.293 \mu \mathrm{m}$, to search for the presence

15 Hence, even if we underestimated the error in our $V$-band photometry (see $\S$ by an order of magnitude, the resulting propagation of error would not significantly affect the size of this discrepancy. 
of features from the $12 \mathrm{CO} 2-0$ bandhead. Such features are very weak for mid-G-type stars, and absent for stars with spectral types earlier than G0. Hence, the detection of such features would indicate the presence of a cool stellar photosphere, as predicted by our blend scenario.

We used a 3-pixel-wide slit, which yields a spectral resolution of approximately 25,000 . We gathered two $4-$ minute exposures, between which we nodded along the slit by roughly $5^{\prime \prime}$. We differenced the two exposures to subtract the sky emission and any pixel-dependent detector bias. We extracted the order spanning the location of the $12 \mathrm{CO} 2-0$ bandhead by summing over a 15-pixel-wide band centered on the peak of the instrumental profile. A small number of values in the extracted 1-dimensional spectrum were corrupted due to bad pixels in the infrared detector. We replaced these values (30 out of 1024 pixels) by interpolation. This region contains a large number of telluric methane features. We produced a model of these features by modifying the electronic version of KPNO/FTS telluric spectrum (Livingston \& Wallace 1991) for airmass, wavelengthsolution, and instrumental point-spread function. Dividing our extracted spectrum by this model yields the stellar spectrum corrected for telluric absorption.

In Figure 8 we plot the resulting spectrum, as well as a spectrum of the nearby M3V star GJ 725A (which shows very prominent $\mathrm{CO}$ features) for comparison. The relative intensity between the individual CO features at 2.3 um does not differ for these two types of stars, although the overall amplitude of the features is reduced for hotter temperatures. We did not attempt to match the spectral type of the NIRSPEC data quantitatively, since our primary goal is to exclude the planetary hypothesis. The spectrum of GSC 03885-00829 clearly shows the $12 \mathrm{CO} 2$ 0 bandhead near $2.293 \mu \mathrm{m}$; the relative depth of the band head is approximately $6 \%$. The detection of this feature confirms the presence of a cool stellar photosphere. Also, for the late $\mathrm{K}$ dwarfs of our model, the $12 \mathrm{CO} 2-0$ band head has a depth of approximately $30 \%$, and the $\mathrm{K}$ dwarfs in our blend model contribute $25 \%$ of the $K$ band flux from the system. Hence the expective depth of this band head in our spectrum is $\sim 7.5 \%$, in rough agreement with the observed relative depth. Thus we interpret this feature as originating in the photospheres of the $\mathrm{K}$-stars of the binary.

The heliocentric Julian Day at mid-exposure was HJD 2453597.83591, which corresponds to an orbital phase of 0.09 , at which point the expected velocity separation between the two $\mathrm{K}$-stars is $89.5 \mathrm{~km} \mathrm{~s}^{-1}$. The $\mathrm{K}$-stars are likely tidally-locked and hence their spectral features will have a $v \sin i=12 \mathrm{~km} \mathrm{~s}^{-1}$. Since this is smaller than the predicted velocity separation at the time of the exposure, we might expect to resolve the individual components of the observed $12 \mathrm{CO} 2-0$ feature from the two $\mathrm{K}$-stars of the binary. And indeed the band head in our spectrum shows two clear peaks of similar depth, with a velocity separation similar to the predicted separation of the $K$ dwarfs. A more careful analysis of this spectrum (using for example the two-dimensional cross-correlation algorithm TODCOR; Zucker \& Mazeh 1994) should recover the components more precisely. However, for the purposes of this paper, it is enough to identify the presence of the light from an eclipsing $K$ binary system in our spectrum, which rules out the transiting planet hypoth- esis.

\section{DISCUSSION}

The difficult task of eliminating any contamination resulting in a false transit signal is the primary challenge currently facing wide field transit surveys. Developing this experience will not only enable us to make firm detections of transiting Jupiters, but will be extremely valuable as we search for Earth-sized planets outside our solar system with NASA's Kepler mission. As with the ground-based wide-field surveys, the challenge with Kepler will not only be obtaining the photometric precision necessary to observe these minute signals, but rejecting all other possible causes of these eclipses as well.

We have presented here one of our disappointments: a candidate that passed all of our initial photometric and spectroscopic tests, but was later shown to be a result of contaminated light from an eclipsing binary. As such, it highlights the difficulty in rejecting all false positives from a transit survey. As the components of this eclipsing binary are both faint $\mathrm{K}$ dwarfs, the resultant radial velocity variations in the light blended with that from the nearby $\mathrm{F}$ star are not detectable. Also, the resultant color dependence of the blended light curve is small, though observable, and the system displays a color redder than that of an isolated $\mathrm{F}$ star.

The TrES survey readily produces on the order of 10 candidate transiting planets from each selected field of view with 15,000-25,000 stars, the majority of these candidates proving to be false positives. Some of these will mimic the expected properties of a planetary system quite closely. Based on our experience of frequent blends, where the telltale indicators of the stellar components are often masked, spectroscopic and even multi-color photometric follow-up is insufficient to confirm the planetary nature of a candidate. An attempt must be made to interpret the observations as those of a blended eclipsing binary, and this interpretation rejected only if the observations are not in agreement. Having meticulously examined the evidence, we can commit to obtain the radial velocity orbit of this firm candidate through high resolution spectroscopy with a high signal-to-noise ratio. It should be emphasized that determining the mass of the candidate from such observations is a necessary step in identifying a transiting planet. The methods to reject astrophysical false positives presented here and by other authors cannot be used to confirm the planetary nature of a candidate; rather they increase the yield of planets from the resource-intensive high-dispersion spectroscopy required for such a confirmation.

FTOD and DC thank Lynne Hillenbrand for her advice and continuing support of this thesis work. We thank the anonymous referee for several constructive comments. We thank Travis Metcalfe, Nicholas Nell, Katherine Brown, Courtney Hoskins, and William Kent for their able assistance during our observations of GSC 03885-00829 with the Sommers Bausch Observatory $24^{\prime \prime}$ telescope. We are grateful for the help of Jose Manuel Almenara and Breezy Ocaña with the observations made using the IAC-80 telescope (operated by the Instituto de Astrofísica de Canarias at the Observatorio del Teide). We also thank Russell White 
for his assistance in gathering our NIRSPEC observations. The authors wish to recognize and acknowledge the very significant cultural role and reverence that the summit of Mauna Kea has always had within the indigenous Hawaiian community. We are most fortunate to have the opportunity to conduct observations from this mountain. This material is based upon work supported by the National Aeronautics and Space Administration under grant NNG05GJ29G, issued through the Origins of Solar Systems Program. GT acknowledges partial support for this work from NASA Origins grant NNG04LG89G. We acknowledge support for this work from NASA's Kepler mission. This research has made use of the SIMBAD database, operated at CDS, Strasbourg, France, and NASA's Astrophysics Data System Bibliographic Services. This publication also utilizes data products from the Two Micron All Sky Survey, which is a joint project of the University of Massachusetts and the Infrared Processing and Analysis Center/California Institute of Technology, funded by the National Aeronautics and Space Administration and the National Science Foundation. This research has made use of the USNOFS Image and Catalogue Archive ${ }^{16}$ operated by the United States Naval Observatory, Flagstaff Station. Facilities: FLWO:1.2m, Hall

16 http://www.nofs.navy.mil/data/f chpix/

\section{REFERENCES}

Alard, C. 2000, A\&AS, 144, 363

Alonso, R., et al. 2004a, ApJ, 613, L153

Alonso, R., Deeg, H. J., Brown, T. M., \& Belmonte, J. A. 2004b, Astron. Nachr., 325, 594

Bakos, G. Á., Lázár, J., Papp, I., Sári, P., \& Green, E. M. 2002, PASP, 114, 974

Baraffe, I., Chabrier, G., Allard, F., \& Hauschildt, P. H. 1998, A\&A, 337, 403

Bessell, M. S., \& Brett, J. M. 1988, PASP, 100, 1134

Boss, A. P. 1997, Science, 276, 1836

Brown, T. M. 2003, ApJ, 593, L125

Carpenter, J. M. 2001, AJ, 121, 2851

Chabrier, G., Baraffe, I., Allard, F., \& Hauschildt, P. H. 2005 , in ASP Conf. Ser. : Resolved Stellar Populations, ed. D. VallsGabaud \& M. Chavez, in press (astro-ph/0509798)

Charbonneau, D., et al. 2005, ApJ, 626, 523

Charbonneau, D., Brown, T. M., Dunham, E. W., Latham, D. W. Looper, D. L., \& Mandushev, G. 2004, in AIP Conf. Proc. 713 The Search for Other Worlds, 151

Charbonneau, D., Brown, T. M., Latham, D. W., \& Mayor, M. 2000, ApJ, 529, L45

Charbonneau, D., Brown, T. M., Noyes, R. W., \& Gilliland, R. L. 2002, ApJ, 568, 377

Charbonneau, D., et al. 2006, ApJ, 636, 445

Creevey, O. L., et al. 2005, ApJ, 625, L127

Cutri, R. M., et al. 2003, VizieR Online Data Catalog, 2246, 0

Deeg, H. J., \& Doyle, L. R. 2001, in Third Workshop on Photometry, ed. W. J. Borucki \& L. E. Lasher, 85

Delfosse, X., Forveille, T., Ségransan, D., Beuzit, J.-L., Udry, S. Perrier, C., \& Mayor, M. 2000, A\&A, 364, 217

Deming, D., Seager, S., Richardson, L. J., \& Harrington, J. 2005 Nature, 434, 740

Dunham, E. W., Mandushev, G. I., Taylor, B. W., \& Oetiker, B. 2004, PASP, 116, 1072

Girardi, L., Bressan, A., Bertelli, G., \& Chiosi, C. 2000, A\&AS, 141,371

Henry, G. W., Marcy, G. W., Butler, R. P., \& Vogt, S. S. 2000 ApJ, 529, L41

Høg, E., et al. 2000, A\&A, 355, L27

Kovács, G., Zucker, S., \& Mazeh, T. 2002, A\&A, 391, 369
Kurucz, R. 1993, ATLAS9 Stellar Atmosphere Programs and 2 $\mathrm{km} / \mathrm{s}$ grid, Kurucz CD-ROM No. 13 (Cambridge: SAO)

Landolt, A. U. 1992, AJ, 104, 340

Latham, D. W. 1992, in ASP Conf. Ser. 32: IAU Colloq. 135: Complementary Approaches to Double and Multiple Star Research, 110

Latham, D. W. 2003, in ASP Conf. Ser. 294: Scientific Frontiers in Research on Extrasolar Planets, 409

Laughlin, G., Wolf, A., Vanmunster, T., Bodenheimer, P., Fischer, D., Marcy, G., Butler, P., \& Vogt, S. 2005, ApJ, 621, 1072

Leggett, S. K. 1992, ApJS, 82, 351

Livingston, W., \& Wallace, L. 1991, An atlas of the solar spectrum in the infrared from 1850 to $9000 \mathrm{~cm}-1$ (1.1 to 5.4 micrometer) (NSO Technical Report 91-001; Tucson: NSO)

Mandushev, G., et al. 2005, ApJ, 621, 1061

Monet, D. G., et al. 2003, AJ, 125, 984

Pollack, J. B. 1984, ARA\&A, 22, 389

Pollack, J. B., Hubickyj, O., Bodenheimer, P., Lissauer, J. J., Podolak, M., \& Greenzweig, Y. 1996, Icarus, 124, 62

Sato, B., et al. 2005, ApJ, 633, 465

Stetson, P. B. 1987, PASP, 99, 191

Stetson, P. B. 1992, in ASP Conf. Ser. 25: Astronomical Data Analysis Software and Systems I, ed. D. M. Worrall, C. Biemesderfer, \& J. Barnes, 297

Street, R. A., et al. 2003, in ASP Conf. Ser. 294: Scientific Frontiers in Research on Extrasolar Planets, 405

Struve, O. 1952, Observatory, 72, 199

Tody, D. 1993, in ASP Conf. Ser. 52: Astronomical Data Analysis Software and Systems II, ed. R. J. Hanisch, R. J. V. Brissenden, \& J. Barnes, 173

Torres, G., Konacki, M., Sasselov, D. D., \& Jha, S. 2004a, ApJ, 609, 1071

Torres, G., Konacki, M., Sasselov, D. D., \& Jha, S. 2004b, ApJ, 614,979

Vidal-Madjar, A., Lecavelier des Etangs, A., Désert, J.-M., Ballester, G. E., Ferlet, R., Hébrard, G., \& Mayor, M. 2003, Nature, 422, 143

Zucker, S., \& Mazeh, T. 1994, ApJ, 420, 806 
TABLE 1

DATA FOR GSC 03885-00829

\begin{tabular}{|c|c|}
\hline Parameter & Value \\
\hline $\begin{array}{ll}\text { R.A. } & \text { (J2000) } \\
\text { Decl. } & (\mathrm{J} 2000) \\
\text { GSC } & \\
\text { 2MASS } & \end{array}$ & $\begin{array}{c}16^{\mathrm{h}} 52^{\mathrm{m}} 33^{\mathrm{s}} \cdot 7 \\
+57^{\circ} 58^{\prime} 27^{\prime \prime} \\
03885-00829 \\
16523368+5758262\end{array}$ \\
\hline $\begin{array}{lc}V^{\mathrm{a}} & (\mathrm{mag}) \\
B-V^{\mathrm{a}} & (\mathrm{mag}) \\
V-R_{\mathrm{C}} & (\mathrm{mag}) \\
V-I_{\mathrm{C}}{ }^{\mathrm{a}} & (\mathrm{mag}) \\
J \mathrm{~b} & (\mathrm{mag}) \\
J-H^{\mathrm{b}} & (\mathrm{mag}) \\
J-K_{s} \mathrm{~b} & (\mathrm{mag})\end{array}$ & $\begin{aligned} 10.465 & \pm 0.001 \\
0.612 & \pm 0.001 \\
0.371 & \pm 0.003 \\
0.743 & \pm 0.001 \\
9.197 & \pm 0.018 \\
0.337 & \pm 0.023 \\
0.440 & \pm 0.026\end{aligned}$ \\
\hline $\begin{array}{lr}\text { Period }^{\mathrm{c}} & (\mathrm{d}) \\
T_{2} \mathrm{~d} & (\mathrm{HJD}) \\
\text { Depth } & (r \text { mag }) \\
\end{array}$ & $\begin{aligned} 2.88244 & \pm 0.00046 \\
2453529.833 & \pm 0.009 \\
0.006 & \pm 0.003\end{aligned}$ \\
\hline \multicolumn{2}{|c|}{$\begin{array}{l}\text { aSee } \$ \text { for a discussion of errors. } \\
{ }^{\mathrm{b}} \text { From the 2MASS Catalog (Cutri et al. 2003). } \\
{ }^{\mathrm{c}} \text { The period of the suspected candidate planet } \\
\text { was } 1.44122 \pm 0 \text { d } 00023 \text {. } \\
{ }^{\mathrm{d}} \text { The time of the secondary eclipse of the binary, } \\
\text { and the central transit time of the candidate. }\end{array}$} \\
\hline
\end{tabular}

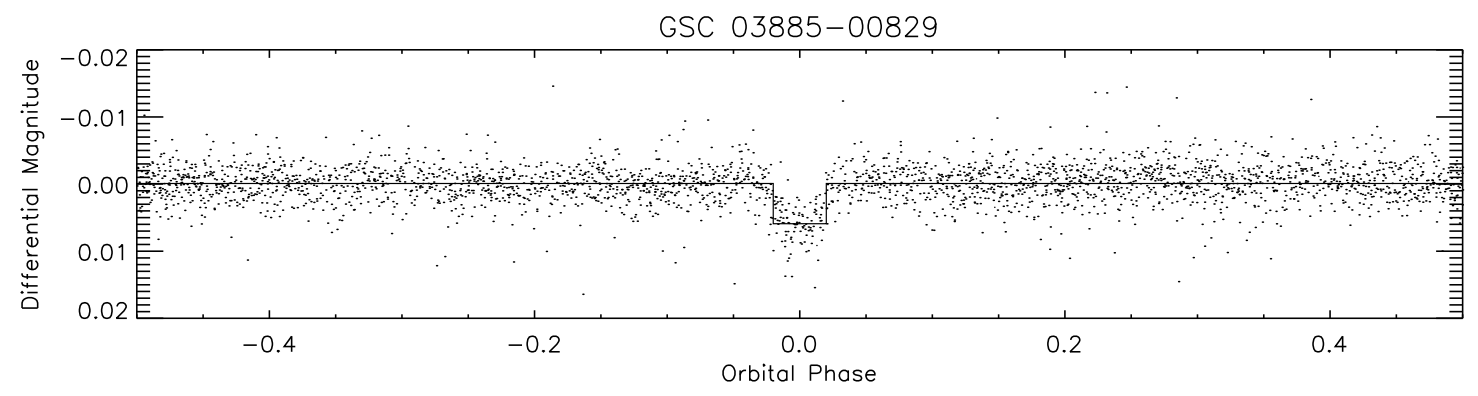

FIG. 1.- Binned TrES $r$ light curve of GSC 03885-00829, folded with the photometric period of 1.441 days computed using the Box-fitting Least Squares algorithm. Overlaid is the corresponding box transit model. 

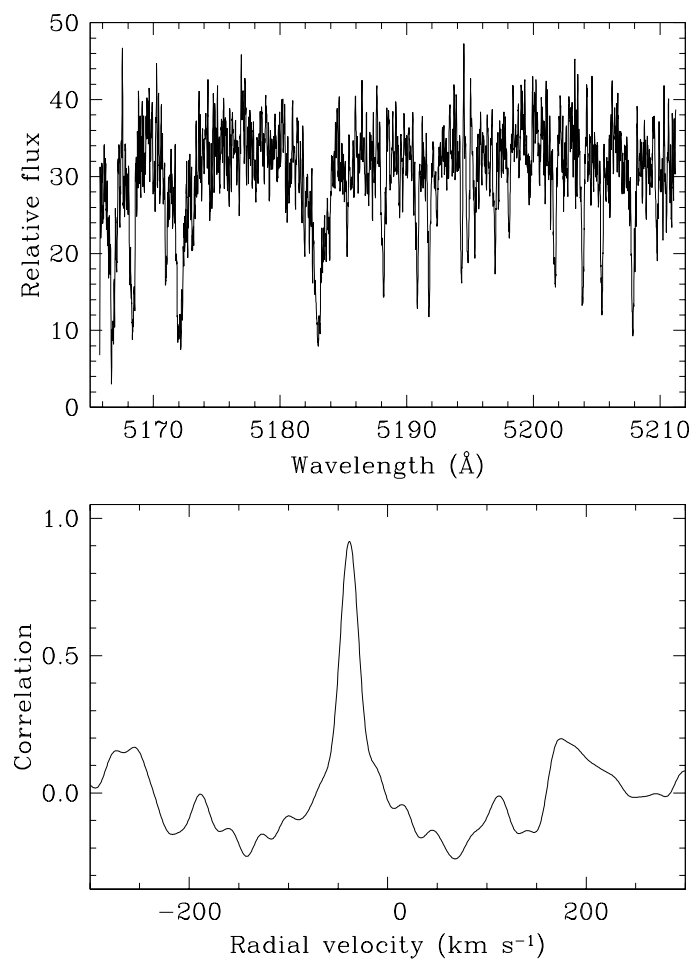

FIG. 2. - Sample spectrum of GSC 03885-00829 (which includes the Mg I b triplet) and the corresponding cross-correlation function.

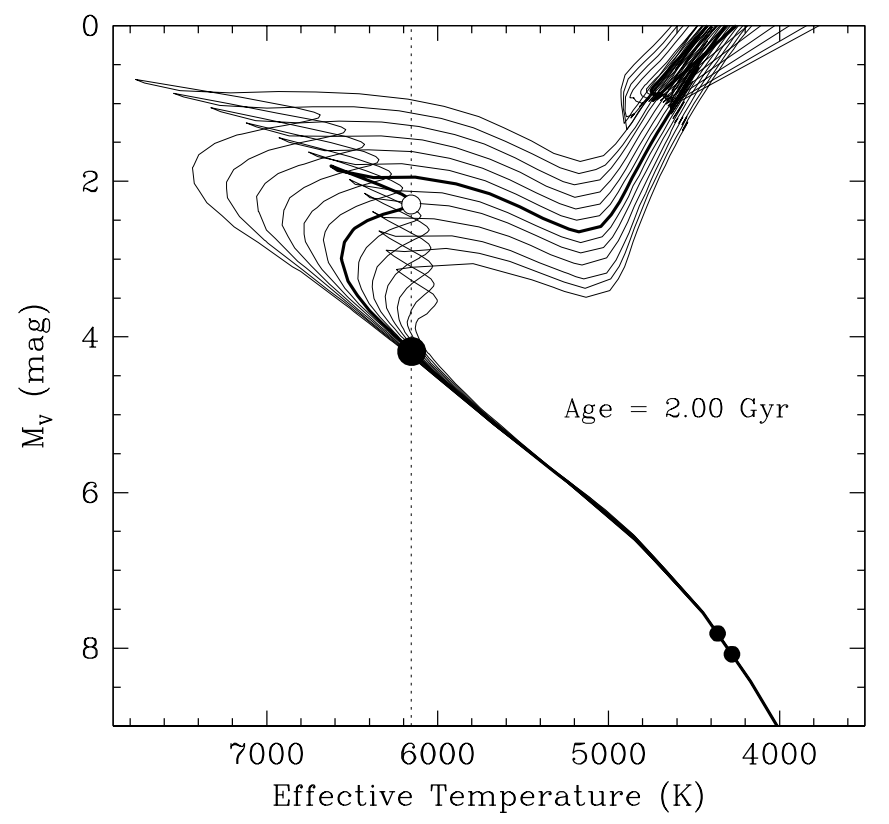

FIG. 3.- Girardi et al. (2000) model isochrones for solar metallicity and ages ranging from 1 to 4 Gyr. The open circle and large filled circle represent two main-sequence stars of the same effective temperature as our candidate GSC 03885-00829 $\left(T_{\text {eff }}=6150 \mathrm{~K}\right)$, but different degrees of evolution. They are shown on the 2 Gyr isochrone (heavy line), which maximizes the difference in brightness at this temperature. The corresponding radii differ by a factor of 2.4. We must therefore constrain the evolution of our candidate using spectroscopy before we can accurately estimate its radius and hence the size of any transiting companion. The three filled circles show the location of each of the three members of our final blend model for this candidate on the 2 Gyr isochrone. The bright primary has a mass of $1.15 \mathrm{M}_{\odot}$, and the binary component masses are $0.67 \mathrm{M}_{\odot}$ and $0.64 \mathrm{M}_{\odot}$. 

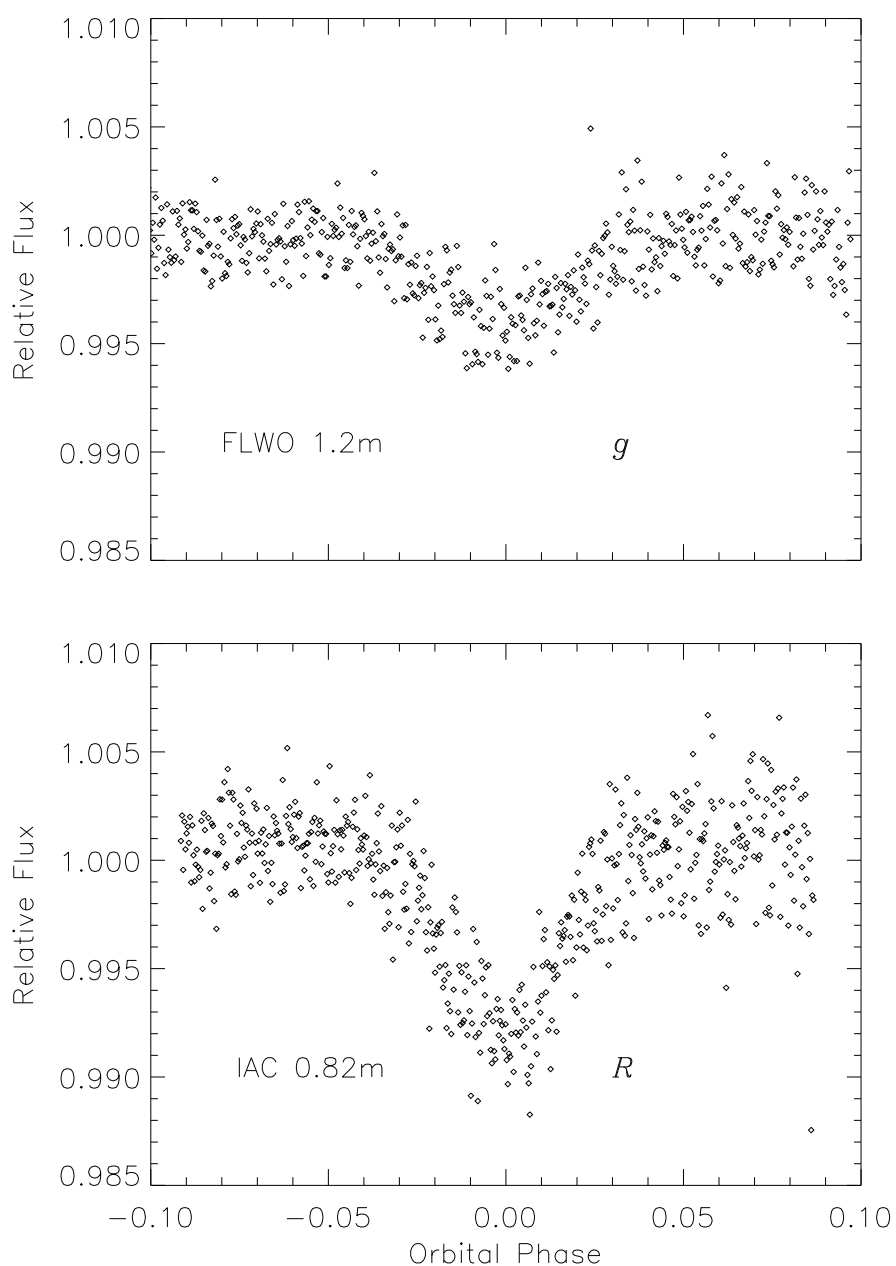

FIG. 4.- Our follow-up photometry of GSC 03885-00829 near the predicted time of transit. The observations were folded using the orbital ephemeris of the planet (see Table 1). Each plot is labelled with the corresponding telescope (see text), and the filter bandpass. The predicted transit events were observed, but an increase in eclipse depth with increasing wavelength is apparent.

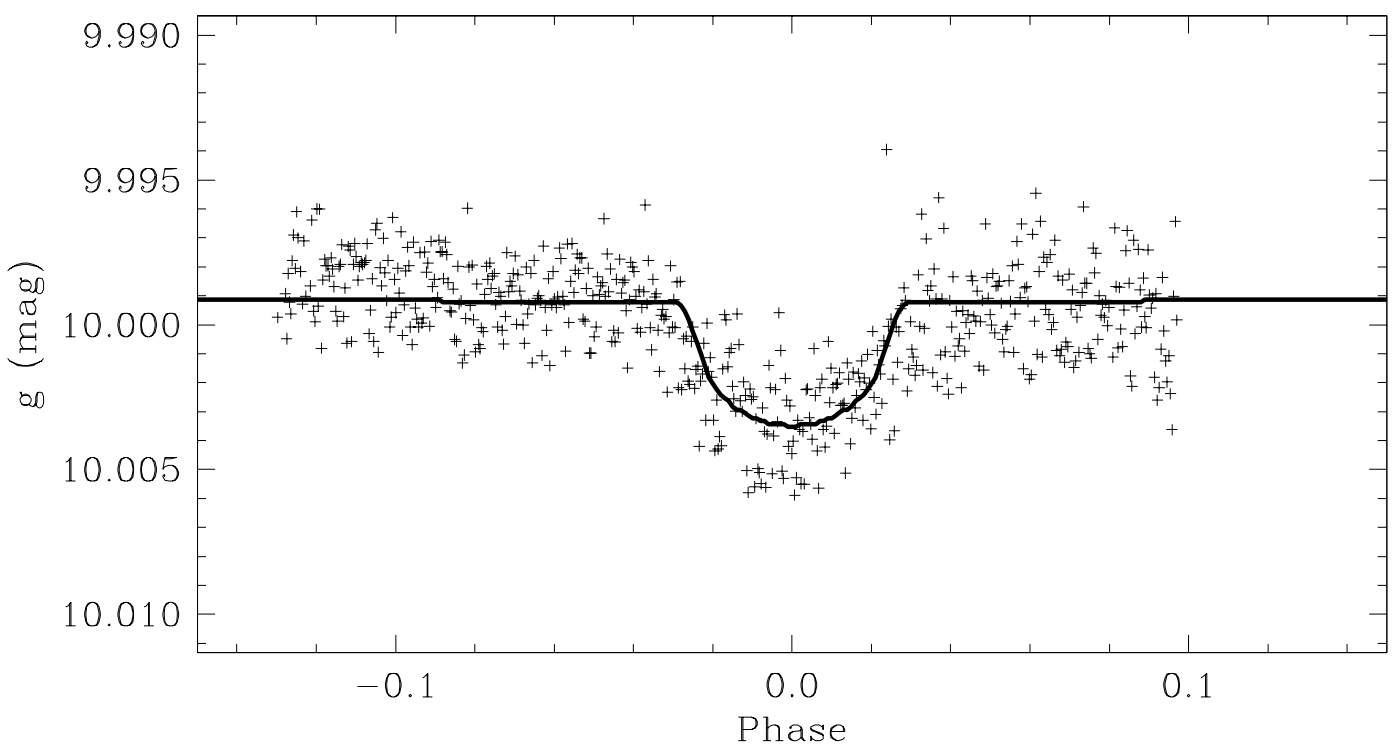

FIG. 5.- Binned $g$-band observations of GSC 03885-00829 obtained with the FLWO $1.2 \mathrm{~m}$ telescope, folded with the BLS period of 1.441 days. Superimposed is the theoretical light curve from a blend model consisting of the bright $\mathrm{F}$ star and a $\mathrm{K}+\mathrm{M}$ dwarf eclipsing binary (see text). Although the depth of the transit is well fit, the predicted duration is slightly shorter than observed. 

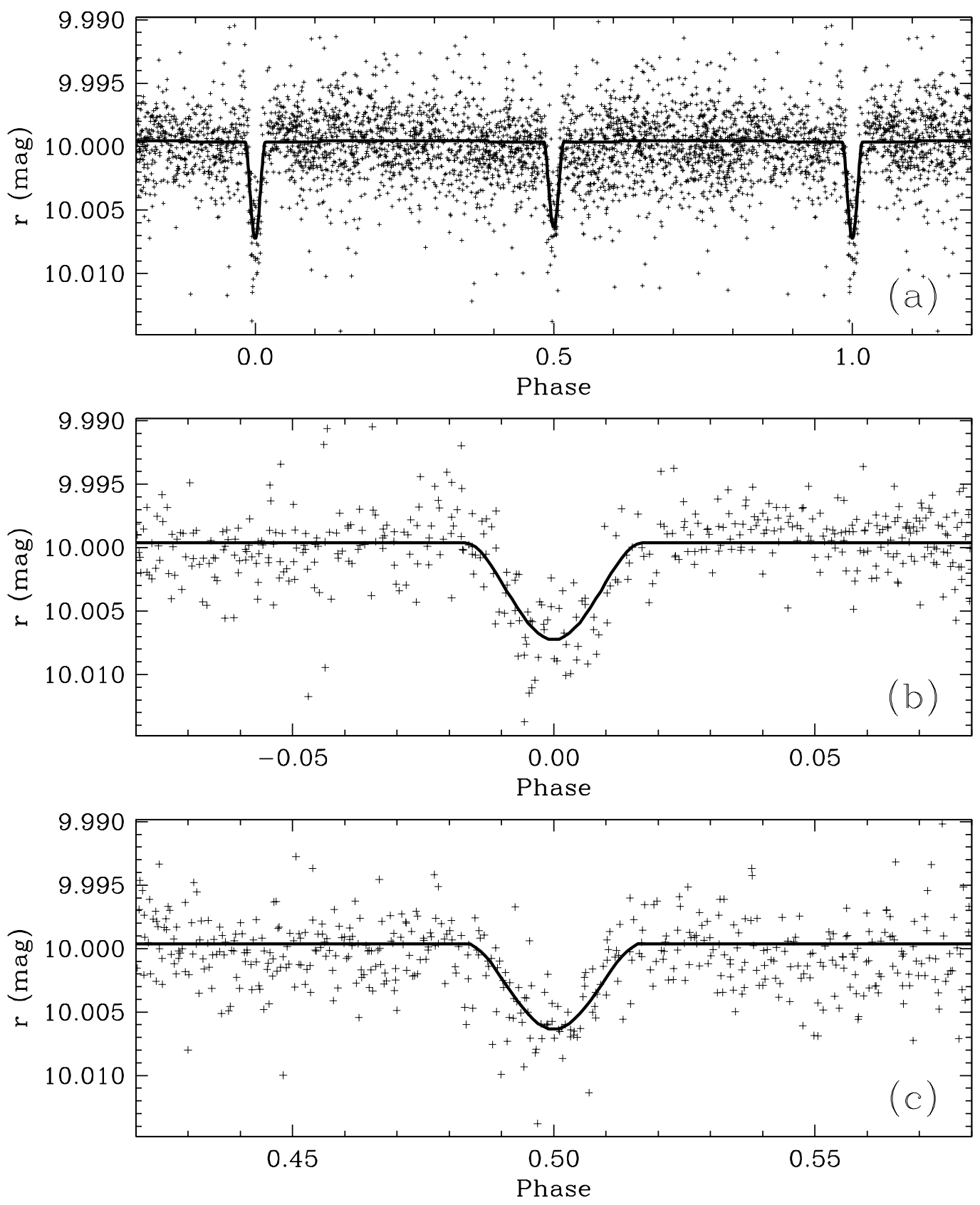

FIG. 6. - TrES $r$-band photometry of GSC 03885-00829, folded using a period twice that of our candidate planet (i.e., $2 \times 1.441$ days). (a) Best-fit blend model consisting of the bright $\mathrm{F}$ star and a pair of eclipsing $\mathrm{K}$ dwarfs (see text). The theoretical curve indicates a small difference in depth between the primary and secondary eclipse; (b) Enlargement around the primary eclipse; (c) Enlargement around the secondary eclipse. The observed duration of the eclipses is well reproduced by the model. 


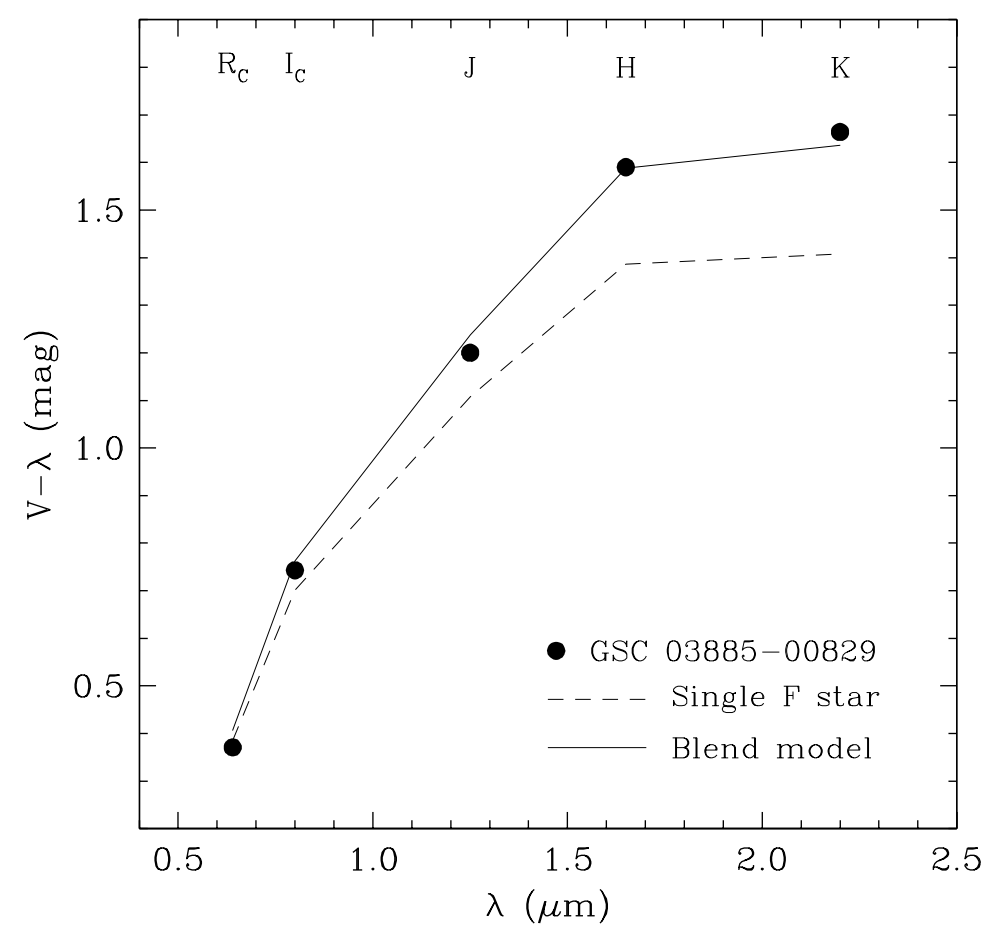

FIG. 7. - Near infrared colors measured for GSC 03885-00829 (dots) compared with the theoretical colors of a single F star (dashed line) and the colors from our blend model (solid line; combined light of three stars). The latter is seen to reproduce the observed colors well.

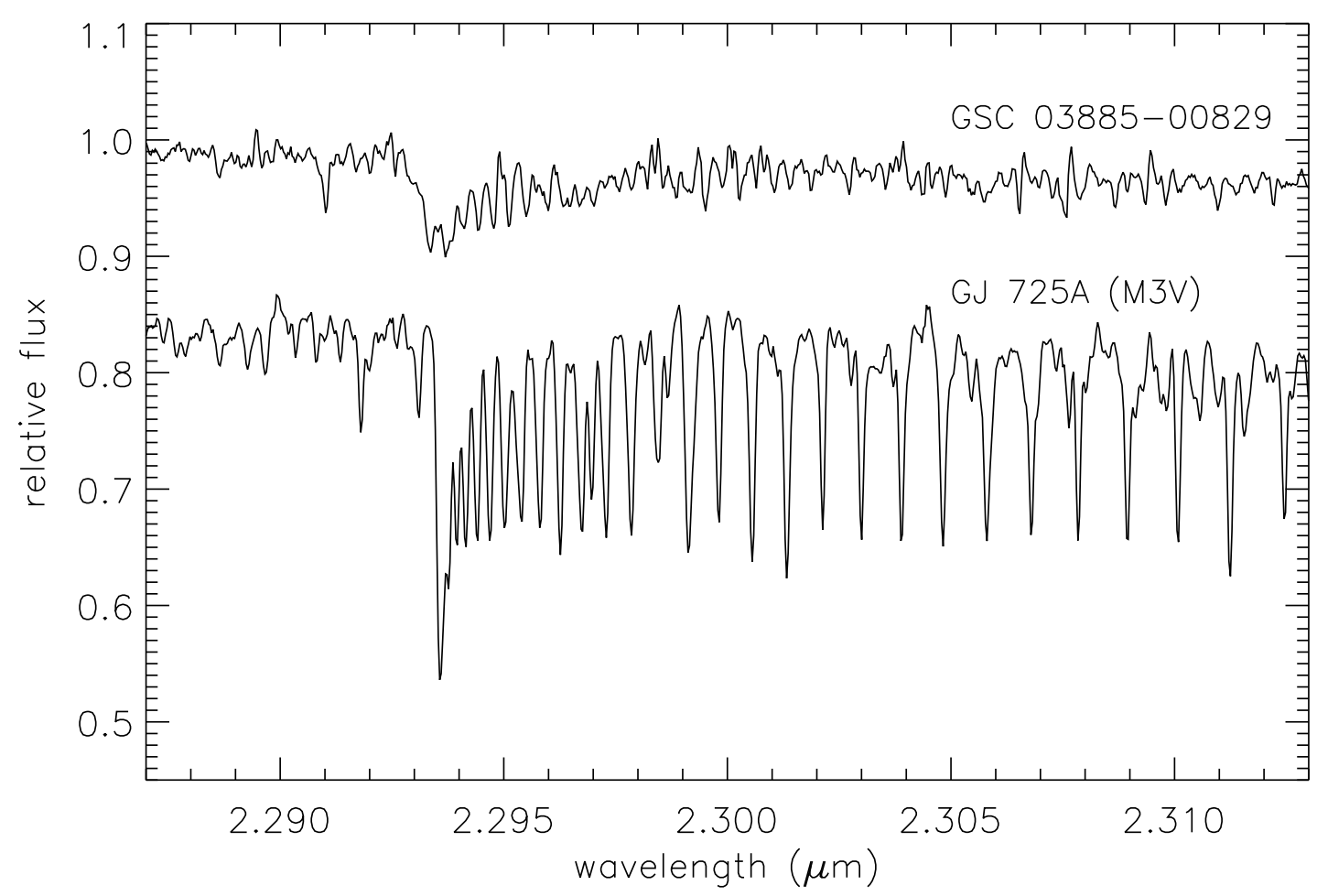

FIG. 8.- K-band spectra of GSC 03885-00829 and a nearby M3V star (GJ 725A), obtained with the NIRSPEC spectrograph at the Keck Observatory. The M dwarf spectrum, with strong CO features, is shown for comparison with our target spectrum, which displays the $12 \mathrm{CO} 2-0$ band head at approximately $2.293 \mu \mathrm{m}$. The spectrum must therefore include the light of a star with a spectral type later than the F dwarf target star, such as the K dwarfs of our blend model. The presence of the CO feature thus rules out the possibility of a planetary companion. 